TRANSACTIONS OF THE

AMERICAN MATHEMATICAL SOCIETY

Volume 352, Number 9, Pages 4237-4256

S 0002-9947(00)02456-9

Article electronically published on March 16, 2000

\title{
A PALAIS-SMALE APPROACH TO PROBLEMS IN ESTEBAN-LIONS DOMAINS WITH HOLES
}

\author{
HWAI-CHIUAN WANG
}

\begin{abstract}
Let $\Omega \subset \mathbb{R}^{N}$ be the upper half strip with a hole. In this paper, we show there exists a positive higher energy solution of semilinear elliptic equations in $\Omega$ and describe the dynamic systems of solutions of equation (1) in various $\Omega$. We also show there exist at least two positive solutions of perturbed semilinear elliptic equations in $\Omega$.
\end{abstract}

\section{INTRODUCTION}

In this paper, we answer affirmatively the Berestycki conjecture by proving that there is a solution of equation (1) in an upper half strip with a hole $\Omega$. Suppose that the solution of equation (1) in an infinite strip $\mathbb{S}$ is unique. Then we describe the dynamic systems of solutions of equation (1) in various $\Omega$ and prove the multiplicity of solutions in perturbed equations (2).

Consider the equation

$$
\begin{cases}-\Delta u+u=u^{p-1} & \text { in } \boldsymbol{\Theta}, \\ u>0 & \text { in } \boldsymbol{\Theta}, \\ u \in H_{0}^{1}(\boldsymbol{\Theta}), & \end{cases}
$$

where $\Theta$ is a domain in $\mathbb{R}^{N}$ and $2<p<\frac{2 N}{N-2}$. The existence of solutions of equation (1) is affected by the properties of the geometry and the topology of the domain $\Theta$.

The existence and nonexistence of solutions of equation (1) have been the focus of a great deal of research in recent years. That equation (1) in a bounded domain admits a solution is a classical result. Gidas-Ni-Nirenberg [12] and Kwong [15] asserted that equation (1) in the whole space $\mathbb{R}^{N}$ admits a "unique" spherically symmetric solution. Therefore, the only interested domains in which equation (1) admits a solution are proper unbounded domains. Such elliptic problems are difficult due to the lack of compactness in an unbounded domain. New analyses are needed to solve such problems. A number of authors have considered the existence and nonexistence of solutions in proper unbounded domains: Lien-Tzeng-Wang [16] and Hsu-Wang [14] asserted that equation (1) in an infinite strip domain admits a ground state solution and Benci-Cerami [2] asserted that equation (1) in an exterior domain admits a higher energy solution.

A proper unbounded domain $\Theta$ in $\mathbb{R}^{N}$ is an Esteban-Lions domain if there is $\chi \in \mathbb{R}^{N},\|\chi\|=1$ such that $n(x) \cdot \chi \geq 0$, and $n(x) \cdot \chi \not \equiv 0$ on $\partial \boldsymbol{\Theta}$, where $n(x)$

Received by the editors July 1, 1996 and, in revised form, May 7, 1998.

1991 Mathematics Subject Classification. Primary 35J20, 35J25.

(C)2000 American Mathematical Society 
denotes the unit outward normal to $\partial \boldsymbol{\Theta}$ at the point $x$. The upper half plane $\mathbb{R}_{+}^{N}$, the epigraph $\Lambda$, the infinite trough $\mathbf{T}$, and the upper half strip $\mathbb{A}$ are Esteban-Lions domains. Esteban-Lions [11] asserted that equation (1) in an Esteban-Lions domain does not admit any solution.

Although these studies have provided much valuable information on the relationship between the existence of solutions of equation (1) and the geometry and the topology of the domain $\boldsymbol{\Theta}$, there is still a great deal to be explored.

The Esteban-Lions paper is the cornerstone and the starting point for studying proper unbounded domains in which equation (1) admits a solution. It is natural to see how the existence of solutions of equation (1) becomes possible in a perturbed Esteban-Lions domain. There are two ways to perturb a domain: one is to add a ball in it and the other is to take out a ball from it.

Lien-Tzeng-Wang 16 and Chen-Lee-Wang 7 asserted the existence of solutions of equation (1) in a perturbed Esteban-Lions domain made by adding a ball in it. We asserted that there exists $s_{0}>0$ such that for the interior flask domain $D_{s}$, which is a perturbed Esteban-Lions domain, equation (1) in $D_{s}$ admits a ground state solution if $s>s_{0}$, but it does not admit any solution if $s<s_{0}$.

As for the existence of solutions of equation (1) in a perturbed Esteban-Lions domain made by taking out a ball from it, that is to say, the Berestycki conjecture: there is a solution of equation (1) in an Esteban-Lions domain with a hole. The Berestycki conjecture has some historical and physical reasons: suppose that there does not exist any solution of an equation in a domain $\boldsymbol{\Theta}$. If we break the symmetry of the domain $\Theta$ by adding a ball in it or by taking out a ball, then the same equation in the perturbed domain admits a solution:

(1) Pohozaev [19] proved that the Dirichlet problem $\Delta u+u^{\frac{N+2}{N-2}}=0$ in a ball does not have any nontrivial solution. But if we take some small ball out, Coron [8] proved that there is a positive solution.

(2) Some turbulence equation in a ball does not admit any nontrivial solution. Lions-Zuazua [17] added a small ball on the boundary to break the symmetry, then he proved that the equation has a nontrivial solution. The phenomenon can be described such that if we add a small material on the surface of a plane, then the turbulence will be controlled.

This paper is organized as follows: Section 2 describes some basic definitions, notation, examples and fundamental properties that will be used later. Section 3 presents the asymptotic behavior of solutions of equation (1) in the infinite strip $\mathbb{S}$ and in the upper half strip with a hole $\Omega$. Section 4 asserts the existence of higher energy solutions of equation (1) in $\Omega$. Section 5 studies the dynamic systems of solutions obtained in Section 4. Section 6 examines multiple solutions of the perturbed equation (2) in $\Omega$ :

$$
\begin{cases}-\Delta u+u=u^{p-1}+f(z) & \text { in } \Omega \\ u>0 & \text { in } \Omega, \\ u \in H_{0}^{1}(\Omega) . & \end{cases}
$$

Our results in this paper are still true for any one of the four known EstebanLions domains above. But for simplicity, we study only the upper half strip with a hole $\Omega$. We also believe that the analyses and the results in this paper will be helpful for studying the existence of solutions of equations in unbounded domains. 
Mathematicians spent ten years proving Lemma 16 in the entire space $\mathbb{R}^{N}$. Berestyski-Lions [3] asserted that there is a ground state solution of equation (1) in $\mathbb{R}^{N}$ and Gidas-Ni-Nirenberg [12] asserted that every solution of equation (1) in $\mathbb{R}^{N}$ is spherically symmetric. Later, Kwong [15] asserted that the spherically symmetric solutions of equation (1) in $\mathbb{R}^{N}$ are unique. In order to prove our existence theorem in Section 4, we need the solution of equation (1) in $\mathbb{S}$ to be unique. In recent years, we have partially established Lemma 16 in $\mathbb{S}$ (see Proposition 28). We assert that there is a ground state solution of equation (1) in $\mathbb{S}$ (see Lien-Tzeng-Wang [16]) and that every solution of equation (1) in $\mathbb{S}$ is spherically symmetric in $x^{\prime}$ and axially symmetric in $x_{N}$ (see Chen-Chen-Wang [6]). That the solution of equation (1) in $\mathbb{S}$ in $\mathbb{R}^{1}$ is unique is easy to prove. Dancer [9] asserted that the solution of equation (1) in $\mathbb{S}$ in $\mathbb{R}^{2}$ is unique. However, in general, the uniqueness of the solution of equation (1) in $\mathbb{S}$ is still open.

\section{Preliminaries}

In this section, we describe definitions, notation, examples, and fundamental properties. We first list four examples of Esteban-Lions domains as follows:

Example 1. The upper half space $\mathbb{R}_{+}^{N}$ is an Esteban-Lions domain.

Example 2. The epigraph $\Lambda=\left\{x \in \mathbb{R}^{N} \mid x_{1}>\psi\left(x_{2}, \cdots, x_{N}\right)\right\}$ is an EstebanLions domain, where $\psi: \mathbb{R}^{N-1} \rightarrow \mathbb{R}$ is of $C^{1}$.

Example 3. The infinite trough $\mathbf{T}=\left(B^{m+1}(0,-4 h) \times \mathbb{R}^{n-1}\right) \cup\left(B^{m} \times \mathbb{R}_{-4 h} \times \mathbb{R}^{n-1}\right)$ is an Esteban-Lions domain, where $N=m+n, m \geq 3, n \geq 1$, and $z=(x, y)$ is the generic point of $\mathbb{R}^{N}$ with $x \in \mathbb{R}^{m}, y \in \mathbb{R}^{n}, B^{m}=\left\{x \in \mathbb{R}^{m}|| x \mid<1\right\}$, for $h>0$, $\mathbb{R}_{-4 h}=\{t \in \mathbb{R} \mid t>-4 h\}, \mathbf{B}^{m+1}(0,-4 h)=\left\{\left(x, y_{1}\right) \in \mathbb{R}^{m} \times \mathbb{R}||\left(x, y_{1}\right)-(0,-4 h) \mid<\right.$ $1\}$.

Example 4. The upper half strip $\mathbb{A}$ is an Esteban-Lions domain, where for $d$, $s, h>0, r, t \in \mathbb{R}$, let

$$
\left\{\begin{array}{l}
B^{N}(z ; s)=\left\{x \in \mathbb{R}^{N}|| x-z \mid<s\right\} \\
\mathbb{S}=\left\{\left(x_{1}, x_{2}, \cdots, x_{N}\right) \in \mathbb{R}^{N} \mid x_{1}^{2}+\cdots+x_{N-1}^{2}<d^{2}\right\}=B^{N-1}(0 ; d) \times \mathbb{R} ; \\
\mathbb{S}_{r}=\left\{\left(x_{1}, x_{2}, \cdots, x_{N}\right) \in \mathbb{S} \mid r<x_{N}\right\} \\
\mathbb{S}_{r, t}=\left\{\left(x_{1}, x_{2}, \cdots, x_{N}\right) \in \mathbb{S} \mid r<x_{N}<t\right\} \\
D_{s}=\mathbb{S}_{0} \cup B^{N}(0 ; s) ; \\
\mathbb{A}=\mathbb{S}_{0} \cup B^{N}(0 ; d) ; \\
\Omega=\Omega_{h}=\mathbb{A} \backslash \overline{B^{N}((0, h) ; d / 2)}, \text { where } h>d .
\end{array}\right.
$$

We say that $\mathbb{S}$ is the infinite strip, $D_{s}$ an interior flask domain, $\mathbb{A}$ the upper half strip, and $\Omega$ an upper half strip with a hole in $\mathbb{R}^{N}$.

We describe some fundamental results as follows: Let $\Theta$ be an unbounded domain in $\mathbb{R}^{N}$. Let the potential operators $a, b: H_{0}^{1}(\Theta) \rightarrow \mathbb{R}$, and let the energy functional $F: H_{0}^{1}(\Theta) \rightarrow \mathbb{R}$ be given by

$$
\left\{\begin{array}{l}
a(u)=\int_{\Theta}\left(|\nabla u|^{2}+u^{2}\right) \\
b(u)=\int_{\Theta}|u|^{p} \\
F(u)=\frac{1}{2} a(u)-\frac{1}{p} b(u) .
\end{array}\right.
$$

In the following, we simply denote Palais-Smale by $(P S)$. 
Definition 5. 1. For $\beta \in \mathbb{R}$, a sequence $\left\{u_{n}\right\} \subset H_{0}^{1}(\Theta)$ is a $(P S)_{\beta}$-sequence for $F$ if $F\left(u_{n}\right) \rightarrow \beta$ and $F^{\prime}\left(u_{n}\right) \rightarrow 0$ strongly as $n \rightarrow \infty$;

2. $\beta \in \mathbb{R}$ is a $(P S)_{\beta}$-value for $F$ if there is a $(P S)_{\beta}$-sequence for $F$;

3. $F$ satisfies the $(P S)_{\beta}$-condition if every $(P S)_{\beta}$-sequence for $F$ contains a convergent subsequence;

4. $F$ satisfies the $(P S)$-condition if, for every $\beta \in \mathbb{R}$, every $(P S)_{\beta}$-sequence for $F$ contains a convergent subsequence.

We examine the relationship among the constrained minimization problem $\alpha_{m}$, the Nehari minimization problem $\alpha_{M}$, and the minimax problem $\alpha_{\Gamma}$, where

$$
\left\{\begin{array}{l}
m=\inf \{a(u) \mid b(u)=1\} ; \\
\alpha_{m}=\left(\frac{1}{2}-\frac{1}{p}\right) m^{p /(p-2)} ; \\
\mathbf{M}=\left\{u \in H_{0}^{1}(\Theta) \backslash\{0\} \mid a(u)=b(u)\right\} ; \\
\alpha_{M}=\inf _{v \in \mathbf{M}} F(v) ; \\
\Gamma=\left\{v \in C\left([0,1], H_{0}^{1}(\Theta)\right) \mid v(0)=0, v(1)=e\right\}, \text { where } F(e)=0 ; \\
\alpha_{\Gamma}=\inf _{v \in \Gamma} \max _{t \in[0,1]} F(v(t)) .
\end{array}\right.
$$

Let $\left\{u_{n}\right\} \subset H_{0}^{1}(\Theta)$ be a $(P S)_{\beta}$-sequence for $F$, then clearly $\beta \geq 0$ and $\left\{u_{n}\right\}$ is bounded in $H_{0}^{1}(\Theta)$.

Lemma 6. $\alpha_{m}, \alpha_{M}$, and $\alpha_{\Gamma}$ are positive (PS)-values for $F$.

Proof. Lien-Tzeng-Wang [16] proved that if $\left\{u_{k}\right\}$ is a minimizing sequence: $a\left(u_{k}\right) \rightarrow$ $m, b\left(u_{k}\right)=1$ for each $k$, then $\left\{v_{k}\right\}$ is a $(P S)_{\alpha_{m}}$-sequence for $F$, where $v_{k}=$ $m^{\frac{1}{p-2}} u_{k}$. Using two different methods, the Ekeland variational principle and the deformation lemma, Brezis-Nirenberg [5] proved that $\alpha_{\Gamma}$ is a $(P S)_{\alpha_{\Gamma}}$-value for $F$. Using the Ekeland variational principle, Stuart [20, Lemma 3.4] proved that there is a $(P S)_{\alpha_{M}}$-sequence for $F$ as well as a minimizing sequence for $\alpha_{M}$. Chen-Lee-Wang 7] generalized Stuart's result by proving that every minimizing sequence for $\alpha_{M}$ is a $(P S)_{\alpha_{M}}$-sequence for $F$.

We investigate the Nehari manifold $\mathbf{M}$ and the zero energy manifold $\mathbf{Z}$ through the unit sphere $\mathbf{U}$, where

$$
\left\{\begin{array}{l}
\mathbf{U}=\left\{u \in H_{0}^{1}(\Theta) \mid\|u\|_{H^{1}}=1\right\} \\
\mathbf{Z}=\left\{u \in H_{0}^{1}(\Theta) \backslash\{0\} \mid \frac{1}{2} a(u)=\frac{1}{p} b(u)\right\}
\end{array}\right.
$$

Note that $\mathbf{M}$ contains every solution of equation (1) in $\Theta$. For $\lambda \geq 0, u \in$ $H_{0}^{1}(\Theta) \backslash\{0\}$, let

$$
h_{u}(\lambda)=F(\lambda u)=\frac{1}{2} \lambda^{2} a(u)-\frac{1}{p} \lambda^{p} b(u) .
$$

Then

$$
\left\{\begin{array}{l}
h_{u}^{\prime}(\lambda)=\lambda a(u)-\lambda^{p-1} b(u) \\
h_{u}^{\prime \prime}(\lambda)=a(u)-(p-1) \lambda^{p-2} b(u) .
\end{array}\right.
$$

From these equalities we can take uniquely $r_{u}, s_{u}$, and $t_{u} \in \mathbb{R}^{+}$such that $0<r_{u}<$ $s_{u}<t_{u}, s_{u} u \in \mathbf{M}, t_{u} u \in \mathbf{Z}$ and

$$
0=h_{u}^{\prime \prime}\left(r_{u}\right)=h_{u}^{\prime}\left(s_{u}\right)=h_{u}\left(t_{u}\right) .
$$


Let $\varphi: \mathbf{U} \rightarrow \mathbf{M}$ and $\psi: \mathbf{U} \rightarrow \mathbf{Z}$ be given by $\varphi(u)=s_{u} u$ and $\psi(u)=t_{u} u$. We apply the implicit function theorem and the Sobolev imbedding theorem to obtain the following lemma:

Lemma 7. $1 . \varphi$ is bijective and of $C^{1,1}$. Moreover, $\mathbf{M}$ is path-connected and there exists a constant $e>0$ such that, for $u \in \mathbf{M},\|u\|_{H^{1}} \geq e$ and $F(u) \geq e$;

2. $\psi$ is bijective and of $C^{1,1}$. Moreover, $\mathbf{Z}$ is path-connected and there exists a constant $e^{\prime}>0$ such that, for $u \in \mathbf{Z},\|u\|_{H^{1}} \geq e^{\prime}$.

In the following two lemmas, we prove that every positive $(P S)_{\beta}$-sequence for $F$ enjoys several interesting properties:

Lemma 8. Let $\left\{u_{n}\right\} \subset H_{0}^{1}(\Theta)$ be a $(P S)_{\beta}$-sequence for $F$ with $\beta>0$. Then there is a sequence $\left\{s_{n}\right\}$ in $\mathbb{R}^{+}$such that $\left\{s_{n} u_{n}\right\} \subset \mathbf{M}$ and $F\left(s_{n} u_{n}\right)=\beta+o(1)$.

Proof. By Lemma 7 , there is a sequence $\left\{s_{n}\right\}$ in $\mathbb{R}^{+}$such that $\left\{s_{n} u_{n}\right\} \subset \mathbf{M}$, thus $s_{n}^{2} a\left(u_{n}\right)=s_{n}^{p} b\left(u_{n}\right)$ for each $n$. That $a\left(u_{n}\right)=b\left(u_{n}\right)+o(1)$ and $F\left(u_{n}\right)=\beta+o(1)$ implies $s_{n}=1+o(1)$. Therefore, $\left|F\left(u_{n}\right)-F\left(s_{n} u_{n}\right)\right|=o(1)$, or $F\left(s_{n} u_{n}\right)=\beta+$ $o(1)$.

Lemma 9. Let $\beta$ be a positive $(P S)_{\beta}$-sequence for $F$. Then we have

1. $\beta \geq \alpha_{m}$;

2. $\beta \geq \alpha_{M}$

3. $\beta \geq \alpha_{\Gamma}$.

Proof. 1. Let $\left\{u_{n}\right\} \subset H_{0}^{1}(\Theta)$ be a $(P S)_{\beta}$-sequence for $F$ with $\beta>0$, we have

$$
\left\{\begin{array}{l}
F\left(u_{n}\right)=\frac{1}{2} a\left(u_{n}\right)-\frac{1}{p} b\left(u_{n}\right)=\beta+o(1), \\
F^{\prime}\left(u_{n}\right)=a\left(u_{n}\right)-b\left(u_{n}\right)=o(1) .
\end{array}\right.
$$

Let $w_{n}=u_{n}\left(b\left(u_{n}\right)\right)^{-1 / p}$, then

$$
b\left(w_{n}\right)=1
$$

and

$$
a\left(w_{n}\right)=a\left(u_{n}\right) b\left(u_{n}\right)^{-2 / p} \geq m .
$$

Thus $a\left(u_{n}\right) \geq m^{p /(p-2)}+o(1)$, or $\beta \geq\left(\frac{1}{2}-\frac{1}{p}\right) m^{p /(p-2)}=\alpha_{m}$.

2. By Lemma 8 , there is a sequence $\left\{s_{n}\right\}$ in $\mathbb{R}^{+}$such that $\left\{s_{n} u_{n}\right\} \subset \mathbf{M}$ and $F\left(s_{n} u_{n}\right)=\beta+o(1)$. Therefore, $\beta \geq \alpha_{M}$.

3. By Lemma 8 , there is a sequence $\left\{s_{n}\right\}$ in $\mathbb{R}^{+}$such that $\left\{s_{n} u_{n}\right\} \subset \mathbf{M}$ and $F\left(s_{n} u_{n}\right)=\beta+o(1)$. By Lemma 7 , there is a sequence $\left\{t_{n}\right\}$ in $\mathbb{R}^{+}$such that $\left\{t_{n} u_{n}\right\}$ in $\mathbf{Z}$. Since the manifold $\mathbf{Z}$ is path-connected, there is a path $\eta_{n}$ in $\mathbf{Z}$ which connects $t_{n} u_{n}$ to $e$. Let $\gamma_{n}^{\prime}$ be the line segment connecting 0 and $t_{n} u_{n}$ and the path $\gamma_{n}=\gamma_{n}^{\prime} \cup \eta_{n}$. We obtain

$$
\alpha_{\Gamma} \leq \max _{0 \leq t \leq 1} F\left(\gamma_{n}(t)\right)=F\left(s_{n} u_{n}\right)=\beta+o(1) .
$$

Thus $\beta \geq \alpha_{\Gamma}$.

By Lemmas 6 and 9, we have

Theorem 10. $\alpha_{m}=\alpha_{M}=\alpha_{\Gamma}$. 
Definition 11. 1. we say that $\alpha(\Theta)=\alpha_{M}$ is the index of the energy functional $F$ in $\Theta$;

2. We say that a solution $u$ of equation (1) is a ground state solution if $F(u)=$ $\alpha(\Theta)$, and is a higher energy solution if $F(u)>\alpha(\Theta)$.

Definition 12. We say that $\Theta$ is a large subdomain of $\mathbb{S}$ if for any positive number $l$, there exist $r, t$ such that $r<t, t-r=l$ and $\mathbb{S}_{r, t} \subset \Theta$.

Example 13. $\mathbb{A}$ and $\mathbb{A} \backslash D$ are large subdomains of $\mathbb{S}$, where $r \in \mathbb{R}$ and $D \subset \mathbb{A}$ is a bounded closed domain.

Using the cut-out function method, we have the following interesting property:

Proposition 14. Let $\Theta$ be a large subdomain of the infinite strip $\mathbb{S}$. Then $\alpha(\Theta)=$ $\alpha(\mathbb{S})$ and the only possible solutions of equation (1) in $\Theta$ are higher energy solutions.

Theorem 15. There exists $s_{0}>0$ such that for the interior flask domains $D_{s}$, which are the perturbed Esteban-Lions domains, equation (1) in $D_{s}$ admits a ground state solution if $s>s_{0}$, but it does not admit any solution if $s<s_{0}$.

Proof. See Lien-Tzeng-Wang [16] and Chen-Lee-Wang [7.

The following two lemmas are useful:

Lemma 16. The only solutions of equation (1) in $\mathbb{R}^{N}$ are ground state solutions. Moreover, the infimum $\alpha\left(\mathbb{R}^{N}\right)$ is achieved by a "unique" positive regular ground state solution $\bar{w} \in H^{1}\left(\mathbb{R}^{N}\right)$ of equation (1) such that $\bar{w}$ is spherically symmetric about a certain point $x_{0}$ in $\mathbb{R}^{N}, \bar{w}^{\prime}(r)<0$ for $r=\left|x-x_{0}\right|$, and

$$
\left\{\begin{array}{l}
\lim _{r \rightarrow \infty} r^{\frac{N-1}{2}} e^{r} \bar{w}(r)=\gamma>0, \\
\lim _{r \rightarrow \infty} r^{\frac{N-1}{2}} e^{r} \bar{w}^{\prime}(r)=-\gamma .
\end{array}\right.
$$

Proof. See Gidas-Ni-Nirenberg [12] and Kwong [15].

Let $\Theta$ be a large subdomain of $\mathbb{S}$. Consider the energy function $F_{f}: H_{0}^{1}(\Theta) \rightarrow \mathbb{R}$ defined by

$$
F_{f}(u)=F(u)-\int_{\Theta} f u .
$$

A $(P S)_{\beta}$-sequence for the perturbed energy functional $F_{f}$ can be defined similarly as the energy functional $F$ in Definition 5 . We have the following decomposition lemma of $(P S)_{\beta}$-sequence for $F_{f}$ :

Lemma 17. Let $\left\{u_{k}\right\}$ be a nonnegative $(P S)_{\beta}$-sequence for $F_{f}$ in $H_{0}^{1}(\Theta)$ :

$$
\begin{cases}F_{f}\left(u_{k}\right)=\beta+o(1) & \text { as } k \rightarrow \infty, \\ F_{f}^{\prime}\left(u_{k}\right)=o(1) & \text { strongly in } H^{-1}(\Theta) .\end{cases}
$$

Then there exist an integer $\ell \geq 0$, sequences $\left\{z_{k}^{i}\right\}$, where $z_{k}^{i}=\left(0, y_{k}^{i}\right) \in \mathbb{S}$ for $1 \leq i \leq \ell$, such that for some subsequence $\left\{u_{k}\right\}$, there are $u^{0} \in H_{0}^{1}(\Theta), u^{0} \geq 0$ in 
$\Theta, u^{i} \in H_{0}^{1}(\mathbb{S}), u^{i}>0$ in $\mathbb{S}, 1 \leq i \leq \ell$, satisfying

$$
\left\{\begin{array}{l}
u_{k}(z)=u^{0}(z)+\left[u^{1}\left(z-z_{k}^{1}\right)+u^{2}\left(z-z_{k}^{2}\right)+\cdots\right. \\
\left.\quad+u^{\ell}\left(z-z_{k}^{\ell}\right)\right]+o(1) \text { strongly in } H_{0}^{1}(\mathbb{S}), \\
-\triangle u^{0}+u^{0}=\left(u^{0}\right)^{p-1}+f(z) \text { in } \Theta, \\
-\triangle u^{i}+u^{i}=\left(u^{i}\right)^{p-1} \text { in } \mathbb{S}, 1 \leq i \leq \ell, \\
F_{f}\left(u_{k}\right)=F_{f}\left(u^{0}\right)+\sum_{i=1}^{\ell} F\left(u^{i}\right)+o(1) \text { as } k \rightarrow \infty, \\
\left|z_{k}^{i}\right| \rightarrow \infty,\left|z_{k}^{i}-z_{k}^{j}\right| \rightarrow \infty \text { for } 1 \leq i \neq j \leq m \text {, as } k \rightarrow \infty .
\end{array}\right.
$$

Proof. See Lien-Tzeng-Wang [16, Theorem 4.1].

\section{Asymptotic Behavior of SOlutions}

Let $\mathbf{X}$ be the infinite strip $\mathbb{S}$, the upper half strip $\mathbb{A}$, or the upper half strip with a hole $\Omega$. Then the domain $\mathbf{X}$ is a $C^{1,1}$ domain and hence we have the Extension Lemma, Embedding Lemma, Interpolation Lemma (see Adams [1 for the proof), and Regularity Lemmas 1-4. For the proof of Regularity Lemma 1, see Brezis-Kato [4]; for the proofs of Regularity Lemmas 2-4, see Gilbarg-Trudinger 13, Theorem $8.8,9.11,9.16]$.

Lemma 18 (Extension). There is a positive constant $c=c(m, p)$ such that for any $u \in W^{m, p}(\mathbf{X}), m \in \mathbb{N}, 1<p<\infty$, there exists some $\bar{u} \in W^{m, p}\left(\mathbb{R}^{N}\right)$ such that $\bar{u}=u$ a.e. in $\mathbf{X}$ and $\|\bar{u}\|_{W^{m, p}\left(\mathbb{R}^{N}\right)} \leq c\|u\|_{W^{m, p}(\mathbf{X})}$.

Lemma 19 (Embedding). There exists the following continuous imbedding

$$
W^{j+m, p}(\mathbf{X}) \rightarrow C^{j, \lambda}(\overline{\mathbf{X}}), \quad 0<\lambda \leq m-\frac{N}{p},
$$

provided $(m-1) p<N<m p$.

Lemma 20 (Interpolation). Given $m \in \mathbb{N}, 1<p<\infty$, there exists a constant $c=c(m, p, N)$ such that for any $0<\varepsilon<1,0 \leq j \leq m-1$, and any $u \in W^{m, p}(\mathbf{X})$

$$
\|u\|_{W^{j, p}(\mathbf{X})} \leq c \varepsilon\|u\|_{W^{m, p}(\mathbf{X})}+\frac{c}{\varepsilon^{j /(m-j)}}\|u\|_{W^{0, p}(\mathbf{X})} .
$$

Lemma 21 (Regularity Lemma 1). Let $g \in L^{2}(\mathbf{X}) \cap L^{\frac{N}{2}}(\mathbf{X})$ and $u$ be a weak solution of

$$
\begin{cases}-\triangle u+u=u^{p-1}+g & \text { in } \mathbf{X}, \\ u>0 & \text { in } \mathbf{X}, \\ u \in H_{0}^{1}(\mathbf{X}) . & \end{cases}
$$

Then $u \in L^{q}(\mathbf{X})$ for $q \in[2, \infty)$.

Lemma 22 (Regularity Lemma 2). Let $\mathbf{X} \subset \mathbb{R}^{N}$ be a domain, $g \in L^{2}(\mathbf{X})$, and let $u \in H^{1}(\mathbf{X})$ be a weak solution of the equation $-\Delta u+u=g$ in $\mathbf{X}$. Then for any subdomain $\mathbf{X}^{\prime} \subset \subset \mathbf{X}$ with $d^{\prime}=\operatorname{dist}\left(\mathbf{X}^{\prime}, \partial \mathbf{X}\right)>0$, we have $u \in H^{2}\left(\mathbf{X}^{\prime}\right)$ and

$$
\|u\|_{H^{2}\left(\mathbf{X}^{\prime}\right)} \leq c\left(\|u\|_{H^{1}(\mathbf{X})}+\|g\|_{L^{2}(\mathbf{X})}\right),
$$

for some $c=c\left(N, \lambda, \vartheta, d^{\prime}\right)$. Furthermore, $u$ satisfies the equation $-\Delta u+u=g$ almost everywhere in $\mathbf{X}$. 
Lemma 23 (Regularity Lemma 3). Let $g \in L^{2}(\mathbf{X})$ and $u \in H_{0}^{1}(\mathbf{X})$ be a weak solution of the equation $-\Delta u+u=g$. Then $u \in H_{0}^{2}(\mathbf{X})$ satisfies

$$
\|u\|_{H^{2}(\mathbf{X})} \leq c\|g\|_{L^{2}(\mathbf{X})},
$$

where $c=c(N, \partial \mathbf{X})$.

Lemma 24 (Regularity Lemma 4). Let $g \in L^{2}(\mathbf{X}) \cap L^{q}(\mathbf{X})$ for some $q \in[2, \infty)$ and $u \in H_{0}^{1}(\mathbf{X})$ be a weak solution of the equation $-\Delta u+u=g$ in $\mathbf{X}$. Then $u \in W^{2, q}(\mathbf{X})$.

By Lemma 24, we obtain the following three results about asymptotic behaviors of solutions:

Lemma 25 (Asymptotic Lemma 1). Let $\mathbb{S}$ be the infinite strip and $0 \leq f(z) \leq$ $c \exp \left(-\sqrt{1+\lambda_{1}+\varepsilon}|y|\right)$, for $z=(x, y) \in \mathbb{S}$. If $u$ is a positive solution of equation (2) in $\mathbb{S}$ :

$$
\begin{cases}-\Delta u+u=u^{p-1}+f(z) & \text { in } \mathbb{S} \\ u>0 & \text { in } \mathbb{S} \\ u \in H_{0}^{1}(\mathbb{S}), & \end{cases}
$$

then $u \in C^{1}(\overline{\mathbb{S}})$ and

$$
\lim _{|y| \rightarrow \infty} u(x, y)=0 \text { uniformly in } x \in B^{N-1}(0 ; d) .
$$

Proof. Let $u$ satisfy

$$
-\triangle u+u=u^{p-1}+f(z) \text { in } \mathbb{S} .
$$

If $0 \leq f(z) \leq c \exp \left(-\sqrt{1+\lambda_{1}+\epsilon}|y|\right)$, for $z=(x, y) \in \mathbb{S}$, then by Lemma 24, we have $u \in W^{2, q}(\mathbb{S})$, for $q \in[2, \infty)$. Therefore, $u \in C^{1}(\overline{\mathbb{S}})$ and there exists a constant $\bar{c}$, such that for $s \in(2,+\infty)$,

$$
\|u\|_{L^{\infty}\left(\Upsilon_{s}\right)} \leq \bar{c}\|u\|_{W^{2, N}\left(\Upsilon_{s}\right)}
$$

where $\boldsymbol{\Upsilon}_{s}=\{z=(x, y) \in \mathbb{S}|| y \mid>s\}$. Since $u \in W^{2, N}(\mathbb{S})$, we get

$$
\lim _{|y| \rightarrow \infty} u(x, y)=0 \quad \text { uniformly in } x \in B^{N-1}(0 ; d) .
$$

Lemma 26 (Asymptotic Lemma 2). Let $\lambda_{1}$ be the first eigenvalue of $-\Delta$ in $B^{N-1}(0 ; d)$ with the Dirichlet problem, let $\phi_{1}$ be the corresponding positive eigenfunction to $\lambda_{1}$, and let $u$ be a solution of equation (1) in $\mathbb{S}$. Then for any $0<\delta<$ $1+\lambda_{1}$ there exist $\gamma>0$ and $\beta>0$ such that

1. $\gamma \phi_{1}(x) e^{-\sqrt{1+\lambda_{1}+\delta}|y|} \leq u(z)$, for $z=(x, y) \in \mathbb{S}$,

2. $u(z) \leq \beta \phi_{1}(x) e^{-\sqrt{1+\lambda_{1}-\delta}|y|}$, for $z=(x, y) \in \mathbb{S}$.

Proof. 1. Let $z_{0} \in \partial \mathbb{S}$ and let $B$ be a small ball in $\mathbb{S}$ such that $z_{0} \in \partial B$. Define for $x \in \mathbf{B}^{N-1}(0 ; d), y \in \mathbb{R}$,

$$
w_{\delta}(z)=\phi_{1}(x) e^{-\sqrt{1+\lambda_{1}+\delta}|y|} \quad \text { for } z \in \mathbb{S} .
$$


Since $w_{\delta}(z)>0, u(z)>0$ for $z \in B, w_{\delta}\left(z_{0}\right)=0, u\left(z_{0}\right)=0$, by the Hopf maximum principle, $\frac{\partial w_{\delta}}{\partial \nu}\left(z_{0}\right)<0, \frac{\partial u}{\partial \nu}\left(z_{0}\right)<0$, where $\nu$ is the outward unit normal vector at $z_{0}$. Thus

$$
\lim _{\substack{z \in \mathbb{S} \\ z \rightarrow z_{0} \\ \text { normally }}} \frac{u(z)}{w_{\delta}(z)}=\frac{\frac{\partial u}{\partial \nu}\left(z_{0}\right)}{\frac{\partial w_{\delta}}{\partial \nu}\left(z_{0}\right)}>0 .
$$

Note that

$$
\frac{u(z)}{w_{\delta}(z)}>0 \quad \text { for } z=(x, y) \in \mathbb{S}
$$

Thus

$$
\frac{u(z)}{w_{\delta}(z)}>0 \quad \text { for } z=(x, y) \text { in } \overline{\mathbb{S}} \text {. }
$$

For $0<\delta<1+\lambda_{1}$, take $R>0$ such that $\delta-\frac{\sqrt{1+\lambda_{1}+\delta}(n-1)}{|y|} \geq 0$ for $|y| \geq R$. Since $w_{\delta}(z)$ and $u(z)$ are in $C^{1}(\overline{\mathbb{S}})$, if set

$$
\gamma=\inf _{\substack{z \in \overline{\mathbb{S}} \\|y| \leq R}} \frac{u(z)}{w_{\delta}(z)}
$$

and $w(z)=\gamma w_{\delta}(z)$ for $z \in \overline{\mathbb{S}}$, then $\gamma>0$ and

$$
w(z) \leq u(z) \text { for } z \in \overline{\mathbb{S}},|y| \leq R .
$$

For $z \in \overline{\mathbb{S}},|y| \geq R$, we have

$$
\begin{aligned}
\Delta(w & -u)(z)-(w-u)(z) \\
& =(\Delta w(z)-w(z))+(-\Delta u(z)+u(z)) \\
& =w(z)\left(\delta-\frac{\sqrt{1+\lambda_{1}+\delta}(n-1)}{|y|}\right)+u^{p-1} \geq 0 .
\end{aligned}
$$

Note that $\lim _{z=(x, y)} w(z)=0$ and $\lim _{z=(x, y)} u(z)=0$ uniformly in $x$. For $k=$ $1,2, \ldots$ we take $L_{k}>R$ such that if $z=(x, y),|y| \geq L_{k}$, then $|w(z)| \leq \frac{1}{2 k}$, $|u(z)| \leq \frac{1}{2 k}$. Let

$$
\mathbb{S}^{k}=\left\{z=(x, y) \in \mathbb{S}|R<| y \mid<L_{k}\right\}
$$

with boundary $\partial \mathbb{S}^{k}=\mathbb{S}_{k}^{1} \cup \mathbb{S}_{k}^{2} \cup S_{k}^{3}$, where

$$
\left\{\begin{array}{l}
\mathbb{S}_{k}^{1}=\{z=(x, y) \in \overline{\mathbb{S}}|| y \mid=R\}, \\
\mathbb{S}_{k}^{2}=\left\{z=(x, y) \in \overline{\mathbb{S}}|x \in \partial \mathbb{S}, R \leq| y \mid \leq L_{k}\right\}, \\
\mathbb{S}_{k}^{3}=\left\{z=(x, y) \in \overline{\mathbb{S}}|| y \mid=L_{k}\right\} .
\end{array}\right.
$$

By the strong maximum principle, for $z \in \mathbb{S}^{k}$,

$$
\begin{aligned}
w(z) & -u(z) \\
& \leq \max \left\{\max _{z \in \mathbb{S}_{k}^{1}}(w-u)^{+}(z), \max _{z \in \mathbb{S}_{k}^{2}}(w-u)^{+}(z), \max _{z \in \mathbb{S}_{k}^{3}}(w-u)^{+}(z)\right\} \\
& \leq \frac{1}{k} .
\end{aligned}
$$


Let $k \rightarrow \infty$, then $w(z) \leq u(z)$ for $z=(x, y), z \in \mathbb{S},|y| \geq R$. Then we have

$$
w(z) \leq u(z) \text { for } z \in \mathbb{S} .
$$

2. For $0<\delta<1+\lambda_{1}$, take $R^{\prime}>0$ such that $u^{p} \leq \frac{\delta}{2} u$ for $|y| \geq R^{\prime}$. Define for $x \in B^{N-1}(0 ; d), y \in \mathbb{R}$,

$$
\left\{\begin{array}{l}
w_{-\delta}(z)=\phi_{1}(x) e^{-\sqrt{1+\lambda_{1}-\delta}|y|} \quad \text { for } z \in \mathbb{S} \\
\frac{1}{\beta}=\inf _{\substack{z \in \mathbb{S} \\
|y| \leq R^{\prime}}} \frac{w_{-\delta}(z)}{u(z)} \\
v(z)=\beta w_{-\delta}(z) \quad \text { for } z \in \overline{\mathbb{S}}
\end{array}\right.
$$

For $z \in \mathbb{S},|y| \geq R^{\prime}$ we have

$$
\begin{aligned}
-\Delta & (u-v)(z)+(u-v)(z) \\
& =(-\Delta u(z)+u(z))+(\Delta v(z)-v(z)) \\
& =u^{p-1}(z)+\left(-\delta-\frac{\sqrt{1+\lambda_{1}-\delta}(n-1)}{|y|}\right) v(z) \\
& \leq \frac{\delta}{2}(u-v)(z)
\end{aligned}
$$

therefore,

$$
-\Delta(u-v)(z)+\left(1-\frac{\delta}{2}\right)(u-v)(z) \leq 0 .
$$

As in part 1, we obtain

$$
u(z) \leq v(z) \text { for } z \in \mathbb{S} .
$$

As a consequence of Asymptotic Lemma 2, we have the following:

Lemma 27 (Asymptotic Lemma 3). Let $\Omega_{1}=\left\{z=(x, y) \in \Omega|| x \mid \leq \frac{2}{3} d, y \geq\right.$ $h+d\}$, let $u$ be a solution of equation (2) in $\Omega$, and let there exist $\epsilon>0, c>0$ such that

$$
0 \leq f(z) \leq c \exp \left(-\sqrt{1+\lambda_{1}+\epsilon}|y|\right), \quad \text { for any } z \in \Omega .
$$

Then, for any $\delta$ with $0<\delta<1+\lambda_{1}$, there exist $c_{1}>0, c_{2}>0$ such that

$$
\begin{cases}c_{1} \exp \left(-\sqrt{1+\lambda_{1}+\delta}|y|\right) \leq u(z), & \text { for all } z \in \Omega_{1}, \\ u(z) \leq c_{2} \exp \left(-\sqrt{1+\lambda_{1}-\delta}|y|\right), & \text { for all } z \in \Omega .\end{cases}
$$

Remark 1. Let $f \equiv 0$, from Lemma 27 , we prove that every positive solution of equation (1) in $\Omega$ has the same asymptotic behavior as in Lemma 27.

\section{Existence RESUlts}

Recall the following two results, one for existence and another for nonexistence:

Proposition 28. Equation (1) in the infinite strip $\mathbb{S}$ admits a ground state solution $\bar{u}$. Furthermore, every solution $u$ of equation (1) in $\mathbb{S}$ is radially symmetric in $x^{\prime}$ and axially symmetric in $x_{N}$; that is to say, $u\left(x^{\prime}, x_{N}-\sigma\right)=u\left(\left|x^{\prime}\right|,\left|x_{N}-\sigma\right|\right)$.

Proof. See Lien-Tzeng-Wang [16, Theorem 4.8] and Chen-Chen-Wang [6].

Proposition 29. Equation (1) in the upper half strip $\mathbb{A}$ does not admit any solution. 
Proof. See Esteban-Lions [11, Theorem I.1].

Let, for $h>d, \mathbf{B}=B^{N}((0, h) ; d / 2)$, and $\Omega=\Omega_{h}=\mathbb{A} \backslash \overline{\mathbf{B}}$ be the upper half strip with a hole. By Proposition 14, there is no ground state solutions of equation (1) in $\Omega$. However, in this section, we prove that there exists a higher energy solution of equation (1) in $\Omega$.

Let $\bar{u}$ be as in Proposition 28, $\bar{h}=(0, h) \in \mathbb{S}$ and $\phi: \mathbb{S} \rightarrow[0,1]$, a $C^{\infty}$ cut-off function such that $0 \leq \phi \leq 1$,

$$
\phi(z)= \begin{cases}0 & \text { for } z \in \mathbf{B} \cup(\mathbb{S} \backslash \mathbb{A}), \\ 1 & \text { for } z \in \mathbb{A} \backslash\left(B^{N}\left((0, h) ; \frac{2}{3} d\right) \cup\{z=(x, y) \in \mathbb{S} \mid y \leq d\}\right),\end{cases}
$$

and

$$
\begin{aligned}
& I=\{0\} \times\left[-\frac{d}{2}, \frac{d}{2}\right], \quad I_{h}=\bar{h}+I, \\
& v_{t}(z)=\phi(z) \bar{u}(z-t-2 \bar{h}) \quad \text { for } z \in \mathbb{S}, \quad t \in I .
\end{aligned}
$$

Then $v_{t} \in H_{0}^{1}(\Omega)$. Furthermore, we have

Lemma 30. For $t \in I$ or $\bar{t} \in I_{h}$, where $\bar{t}=\bar{h}+t$, then

1. $\left\|v_{t}(z)-\bar{u}(z-t-2 \bar{h})\right\|_{L^{p}(\mathbb{S})}=o(1)$ as $h \rightarrow \infty$;

2. $\left\|v_{t}(z)-\bar{u}(z-t-2 \bar{h})\right\|_{H^{1}(\mathbb{S})}=o(1)$ as $h \rightarrow \infty$;

3. $F\left(v_{t}\right)=\alpha(\mathbb{S})+o(1)$ as $h \rightarrow \infty$.

Proof. 1. We have

$$
\begin{aligned}
\left\|v_{t}(z)-\bar{u}(z-t-2 \bar{h})\right\|_{L^{p}(\mathbb{S})}^{p} & =\int_{\mathbb{S}}|\phi(z)-1|^{p}|\bar{u}(z-t-2 \bar{h})|^{p} \\
& \leq \int_{\mathbb{S}_{h+d}^{c}}|\bar{u}(z-t-2 \bar{h})|^{p} \\
& =o(1) \quad \text { as } h \rightarrow \infty .
\end{aligned}
$$

2. We have

$$
\begin{aligned}
\| v_{t}(z) & -\bar{u}(z-t-2 \bar{h}) \|_{H^{1}(\mathbb{S})}^{2} \\
& =\|(\phi(z)-1) \bar{u}(z-t-2 \bar{h})\|_{H^{1}(\mathbb{S})}^{2} \\
& \leq c\left(\frac{1}{d^{2}}+1\right) \int_{\mathbb{S}_{h+d}^{c}}\left(|\nabla \bar{u}(z-t-2 \bar{h})|^{2}+|\bar{u}(z-t-2 \bar{h})|^{2}\right) \\
& =o(1) \quad \text { as } h \rightarrow \infty .
\end{aligned}
$$

3. It follows from (1), (2), Proposition 28, and the following

$$
\begin{aligned}
\alpha(\mathbb{S}) & =F(\bar{u})=\frac{1}{2} a(\bar{u})-\frac{1}{p} b(\bar{u}) \\
& =\frac{1}{2} a\left(v_{t}\right)-\frac{1}{p} b\left(v_{t}\right)+o(1)=F\left(v_{t}\right)+o(1) .
\end{aligned}
$$

From Lemma 30, since $\|\bar{u}\|_{H^{1}(\mathbb{S})}^{2}=\|\bar{u}\|_{L^{p}(\mathbb{S})}^{p}$, we have

$$
\begin{cases}\left\|v_{t}\right\|_{H^{1}(\mathbb{S})}^{2}=\|\bar{u}\|_{H^{1}(\mathbb{S})}^{2}+o(1) & \text { as } h \rightarrow \infty \\ \left\|v_{t}\right\|_{L^{p}(\mathbb{S})}^{p}=\|\bar{u}\|_{L^{p}(\mathbb{S})}^{p}+o(1) & \text { as } h \rightarrow \infty\end{cases}
$$


Therefore, $\left\|v_{t}\right\|_{H^{1}(\mathbb{S})}^{2}=\left\|v_{t}\right\|_{L^{p}(\mathbb{S})}^{p}+o(1)$ as $h \rightarrow \infty$. By Lemma 7, there exists $\lambda_{t}>0$ such that $u_{t}=\lambda_{t} v_{t}$ in $\mathbf{M}:\left\|u_{t}\right\|_{H^{1}(\mathbb{S})}^{2}=\left\|u_{t}\right\|_{L^{p}(\mathbb{S})}^{p}$. Therefore, $\lambda_{t} \rightarrow 1$ as $h \rightarrow \infty$, or $F\left(u_{t}\right)=\alpha(\mathbb{S})+o(1)$ as $h \rightarrow \infty$. For $u \in H_{0}^{1}(\Omega)$, define the center mass function by

$$
j(u)=\|u\|_{L^{p}(\mathbb{S})}^{-p} \int_{\mathbb{S}}\left(\bar{h}+\frac{d}{2} \frac{z}{|z|}\right)|u(x, y)|^{p} d x d y .
$$

Let

$$
\alpha_{0}=\inf \{F(u) \mid u \in \mathbf{M}, u \geq 0, j(u)=\bar{h}\} .
$$

Proposition 31. $\alpha(\mathbb{S})=\alpha(\Omega)<\alpha_{0}$.

Proof. By Proposition 14, $\alpha(\mathbb{S})=\alpha(\Omega)$.

Clearly $\alpha(\mathbb{S}) \leq \alpha_{0}$. Suppose $\alpha(\mathbb{S})=\alpha_{0}$. By Lemma 6 , there is a sequence $\left\{u_{k}\right\}$ in $\mathbf{M}, u_{k} \geq 0, j\left(u_{k}\right)=\bar{h}$ for each $k$, such that

$$
\begin{cases}F\left(u_{k}\right)=\alpha(\mathbb{S})+o(1) & \text { as } k \rightarrow \infty, \\ F^{\prime}\left(u_{k}\right)=o(1) & \text { strongly in } H^{-1}(\Omega) \text { as } k \rightarrow \infty .\end{cases}
$$

By Lemma 17 , there is an unbounded sequence $\left\{\left(0, y_{k}\right)\right\}$ in $\mathbb{S}$ such that

$$
u_{k}(x, y)=\bar{u}\left(x, y-y_{k}\right)+o(1) \quad \text { strongly in } H_{0}^{1}(\mathbb{S}),
$$

where $\bar{u}$ is as in Proposition 28. Assume $\left(\bar{h}+\frac{d}{2} \frac{\left(0, y_{k}\right)}{\left(0, y_{k}\right) \mid}\right)=\varsigma+o(1)$ as $k \rightarrow \infty$, where $\varsigma \in \partial I_{h}$, then by the Lebesgue Dominated Convergence Theorem, we have

$$
\begin{aligned}
\bar{h}=j\left(u_{k}\right) & =\left\|u_{k}\right\|_{L^{p}(\mathbb{S})}^{-p} \int_{\mathbb{S}}\left(\bar{h}+\frac{d}{2} \frac{z}{|z|}\right)\left|u_{k}(x, y)\right|^{p} d x d y \\
& =\|\bar{u}\|_{L^{p}(\mathbb{S})}^{-p} \int_{\mathbb{S}}\left(\bar{h}+\frac{d}{2} \frac{\left(x, y+y_{k}\right)}{\left|\left(x, y+y_{k}\right)\right|}\right)|\bar{u}(x, y)|^{p} d x d y+o(1) \\
& =\varsigma+o(1) \text { as } k \rightarrow \infty,
\end{aligned}
$$

which is a contradiction. Therefore, $\alpha(\mathbb{S})=\alpha(\Omega)<\alpha_{0}$.

Let

$$
\left\{\begin{array}{l}
V=\{u \in \mathbf{M} \mid u \geq 0\} \\
\Gamma=\left\{k: I_{h} \rightarrow V \text { continuous } \mid k(\bar{t})=u_{t} \text { for } t \in \partial I\right\} \\
\alpha_{1}=\inf _{k \in \Gamma} \max _{\bar{t} \in I_{h}} F(k(\bar{t})) .
\end{array}\right.
$$

Proposition 32. There is $h_{0}>0$ such that for $h \geq h_{0}$,

1. $\alpha(\mathbb{S})<F\left(u_{t}\right)<\frac{\alpha_{0}+\alpha(\mathbb{S})}{2}<\alpha_{0}$, for $t \in I$;

2. $\alpha(\mathbb{S})<F\left(u_{t}\right)<2^{\frac{p-2}{p}} \alpha(\mathbb{S})$, for $t \in I$;

3. $\left(j \circ u_{t}, \bar{t}\right)>0$, for $t \in \partial I$.

Proof. 1 and 2 follow from Propositions 14 and 31 and Lemma 30.

3. There are $c_{1}, c_{2}>0$ such that $c_{1} \leq\|\phi(z) \bar{u}(z-t-2 \bar{h})\|_{L^{p}(\mathbb{S})} \leq c_{2}$. For $t \in \partial I$ with $z+t+2 \bar{h} \neq 0$, we have

$$
\begin{gathered}
\left(\frac{z+t+2 \bar{h}}{|z+t+2 \bar{h}|}, t\right)=|z+t+2 \bar{h}|-\frac{1}{|z+t+2 \bar{h}|}(z+t+2 \bar{h}, z+2 \bar{h}) \\
\quad \geq|z+t+2 \bar{h}|-|z+2 \bar{h}| \geq|t|-2|z+2 \bar{h}|=\frac{d}{2}-2|z+2 \bar{h}|
\end{gathered}
$$


Then there are constants $c_{3}, c_{4}, c_{5}, c_{6}, h_{0}>0$ such that for $h \geq h_{0}$

$$
\begin{aligned}
\left(j\left(u_{t}\right), \bar{h}+t\right) & =\left.|| u_{t}(z)\right|_{L^{p}(\mathbb{S})} ^{-p} \int_{\mathbb{S}}\left(\bar{h}+\frac{d}{2} \frac{z}{|z|}, \bar{h}+t\right)\left|u_{t}(z)\right|^{p} d z \\
& =c_{3} \int_{\mathbb{S}}\left(\bar{h}+\frac{d}{2} \frac{z}{|z|}, \bar{h}+t\right)|\phi(z) \bar{u}(z-t-2 \bar{h})|^{p} d z \\
& =c_{3} \int_{\mathbb{S}}\left(\bar{h}+\frac{d}{2} \frac{z+t+2 \bar{h}}{|z+t+2 \bar{h}|}, \bar{h}+t\right)|\phi(z+t+2 \bar{h}) \bar{u}(z)|^{p} d z \\
& \geq c_{3}\left(h^{2} c_{5}-h c_{4} c_{5}-h c_{5}-2 c_{6}-4 h c_{5}\right)>0 \text { as } h \geq h_{0} .
\end{aligned}
$$

where $\int_{\mathbb{S}}|\phi(z+t+2 \bar{h}) \bar{u}(z)|^{p} d z \geq c_{5}$ and $\int_{\mathbb{S}}|z||\phi(z+t+2 \bar{h}) \bar{u}(z)|^{p} d z \geq c_{6}$. By Lemma 26, $c_{4}<\infty$.

Proposition 33. For $h \geq h_{0}$, we have

$$
\alpha(\mathbb{S})<\alpha_{0}=\alpha_{1}<2^{\frac{p-2}{p}} \alpha(\mathbb{S}) .
$$

Proof. We claim that

1. $\alpha_{0}=\alpha_{1}$ For any $k \in \Gamma$, consider the homotopy $H(\lambda, \bar{t}):[0,1] \times I_{h} \rightarrow \mathbb{R}^{N}$ defined by

$$
H(\lambda, \bar{t})=(1-\lambda) j(k(\bar{t}))+\lambda i(\bar{t}),
$$

where $i$ denotes the identity map. Note that $j(k(\bar{t}))=j\left(u_{t}\right)$ for $\bar{t} \in \partial I_{h}$. By Proposition $32(3), H(\lambda, \bar{t}) \neq \bar{h}$ for $\bar{t} \in \partial I_{h}$ and $\lambda \in[0,1]$. Therefore,

$$
\operatorname{deg}\left(j \circ k, I_{h}, \bar{h}\right)=\operatorname{deg}\left(i, I_{h}, \bar{h}\right)=1 .
$$

There exists $t_{0} \in I_{h}$ such that

$$
j\left(k\left(t_{0}\right)\right)=\bar{h}
$$

Hence, for each $k \in \Gamma$,

$$
\begin{aligned}
\alpha_{0} & =\inf \{F(u) \mid u \in \mathbf{M}, u \geq 0, j(u)=\bar{h}\} \\
& \leq F\left(k\left(t_{0}\right)\right) \\
& \leq \max _{\bar{t} \in I_{h}} F(k(\bar{t})) .
\end{aligned}
$$

We have $\alpha_{0} \leq \alpha_{1}$. On the other hand, by Proposition 32 (1), for $t \in I$, we have $u_{t} \in V$ and $F\left(u_{t}\right)<\alpha_{0}$. Thus $\max _{t \in I} F\left(u_{t}\right) \leq \alpha_{0}$, or $\alpha_{1} \leq \alpha_{0}$.

2. $\alpha_{1}<2^{\frac{p-2}{p}} \alpha(\mathbb{S})$ : By Proposition $32(2), F\left(u_{t}\right)<2^{\frac{p-2}{p}} \alpha(\mathbb{S})$ for $t \in I$. Thus

$$
\max _{t \in I} F\left(u_{t}\right)<2^{\frac{p-2}{p}} \alpha(\mathbb{S}) .
$$

We have $\alpha_{1}<2^{\frac{p-2}{p}} \alpha(\mathbb{S})$. By Proposition 32 (1), we have

$$
\alpha(\mathbb{S})<\alpha_{0}=\alpha_{1}<2^{\frac{p-2}{p}} \alpha(\mathbb{S})
$$

Now we are going to assert that there is a higher energy solution of equation (1) in $\Omega$.

Theorem 34. Suppose that the solution of equation (1) in the infinite strip $\mathbb{S}$ is unique up to $y$-translations. There exists $h_{0}>0$ such that if $h \geq h_{0}$, then there is a positive higher energy solution $v$ of equation (1) in the upper half strip with a hole $\Omega$ such that $\alpha(\mathbb{S})<F(v)<2^{\frac{p-2}{p}} \alpha(\mathbb{S})$. 
Proof. Note that $\alpha_{0}=\inf \{F(u) \mid u \in \mathbf{M}, u \geq 0, j(u)=\bar{h}\}$. Take a minimizing sequence $\left\{u_{k}\right\}$ in $\mathbf{M}: F\left(u_{k}\right) \rightarrow \alpha_{0}$ as $k \rightarrow \infty$. By Lemma $6,\left\{u_{k}\right\}$ is a $(P S)_{\alpha_{0}}$ sequence for $F: F\left(u_{k}\right) \rightarrow \alpha_{0}$ and $F^{\prime}\left(u_{k}\right) \rightarrow 0$ as $k \rightarrow \infty$. By Lemma 17, there exist an integer $\ell \geq 0$, sequences $\left\{z_{k}^{i}\right\}$, where $z_{k}^{i}=\left(0, y_{k}^{i}\right) \in \mathbb{S}$ for $1 \leq i \leq \ell$, such that for some subsequence $\left\{u_{k}\right\}$, there are $u^{0} \in H_{0}^{1}(\Omega), u^{0} \geq 0$ in $\Omega, u^{i} \in H_{0}^{1}(\mathbb{S}), u^{i}>0$ in $\mathbb{S}, 1 \leq i \leq \ell$, satisfying

$$
\left\{\begin{array}{l}
u_{k}(z)=u^{0}(z)+\left[u^{1}\left(z-z_{k}^{1}\right)+u^{2}\left(z-z_{k}^{2}\right)+\cdots\right. \\
\left.\quad+u^{\ell}\left(z-z_{k}^{\ell}\right)\right]+o(1) \quad \text { strongly in } H_{0}^{1}(\mathbb{S}), \\
-\triangle u^{0}+u^{0}=\left(u^{0}\right)^{p-1} \quad \text { in } \Omega, \\
-\triangle u^{i}+u^{i}=\left(u^{i}\right)^{p-1} \quad \text { in } \mathbb{S}, 1 \leq i \leq \ell, \\
F\left(u_{k}\right)=F\left(u^{0}\right)+\sum_{i=1}^{\ell} F\left(u^{i}\right)+o(1) \text { as } k \rightarrow \infty .
\end{array}\right.
$$

Suppose that the solution of equation (1) in the infinite strip $\mathbb{S}$ is unique up to $y$-translations and from Proposition 28, we obtain that the $u^{i}$ are the same and $F\left(u^{i}\right)=\alpha(\mathbb{S})$ for $i=1,2, \cdots, l$. Therefore,

$$
\alpha_{0}=F\left(u^{0}\right)+l \alpha(\mathbb{S}) .
$$

Since $\alpha(\mathbb{S})<\alpha_{0}<2^{\frac{p-2}{p}} \alpha(\mathbb{S})$, we conclude that $u^{0}$ is nonzero and $l=0$. Thus there is a positive higher energy solution $v=u^{0}$ of equation (1) in the upper half strip with a hole $\Omega$ such that $\alpha(\mathbb{S})<F(v)=\alpha_{0}<2^{\frac{p-2}{p}} \alpha(\mathbb{S})$.

\section{Dynamic Systems OF SOlutions}

As in Section 4 , for $k=1,2, \cdots$, define $\Omega_{k}=\mathbb{A} \backslash \overline{B^{N}\left((0, h) ; \frac{1}{k}\right)}$, where $h \geq$ $2 h_{0}, \frac{1}{k}<d$, then $\Omega_{k}$ is an increasing sequence such that $\mathbb{A} \backslash\{0\}=\bigcup_{k=1}^{\infty} \Omega_{k}$. By Theorem 34, we have, for each $k$, a positive solution $u_{k} \in H_{0}^{1}\left(\Omega_{k}\right)$ of $-\Delta u_{k}+u_{k}=$ $u_{k}^{p-1}$ in $\Omega_{k}$ satisfying

$$
\alpha(\mathbb{S})<F\left(u_{k}\right)<2^{\frac{p-2}{p}} \alpha(\mathbb{S}) .
$$

Moreover, we have

Lemma 35. If $u_{k} \rightarrow u$ weakly in $H_{0}^{1}(\mathbb{A})$ as $k \rightarrow \infty$, then $u \equiv 0$.

Proof. For $\varphi \in C_{0}^{\infty}(\mathbb{A})$, we have

$$
\int_{\mathbb{A}} u_{k}(-\Delta \varphi+\varphi)=\int_{\mathbb{A}}\left(-\Delta u_{k}+u_{k}\right) \varphi=\int_{\mathbb{A}} u_{k}^{p-1} \varphi .
$$

Let $k \rightarrow \infty$, we obtain

$$
\int_{\mathbb{A}} u(-\Delta \varphi+\varphi)=\int_{\mathbb{A}} u^{p-1} \varphi .
$$

Thus $-\Delta u+u=u^{p-1}$ in $\mathbb{A}$. By Proposition $29, u \equiv 0$, or $u_{k} \rightarrow 0$ weakly in $H_{0}^{1}(\mathbb{A})$ as $k \rightarrow \infty$.

We have the following dynamic systems of solutions $\left\{u_{k}\right\}$ :

Theorem 36. $\left|\nabla u_{k}\right|^{2} d z=c \delta_{0}+o(1)$ for some positive number $c$. 
Proof. Let $u_{k} \rightarrow u$ weakly in $H_{0}^{1}(\mathbb{A})$ as $k \rightarrow \infty, \mu_{k}=\left|\nabla u_{k}\right|^{2} d z=\mu+o(1)$ weak $^{*}$, and $\nu_{k}=\left|u_{k}\right|^{p} d z=\nu+o(1)$ weak $^{*}$, then by the second concentration lemma (see Lions [18, Lemma I.1, p. 24]), there exist $\left\{a_{j}\right\}_{j=1}^{\infty},\left\{b_{j}\right\}_{j=1}^{\infty}$ in $\mathbb{R}^{+}$such that

$$
\begin{aligned}
m^{p /(p-2)}+o(1) & =\left\|u_{k}\right\|_{H^{1}\left(\Omega_{k}\right)}^{2}=\int_{\mathbb{A}} d \mu_{k}=\int_{\mathbb{A}} d \mu+o(1) \\
& \geq\|u\|_{H^{1}\left(\Omega_{k}\right)}^{2}+\sum_{j} a_{j}+o(1) \\
& \geq m\left(\|u\|_{L^{p}}^{2}+\sum_{j} b_{j^{\frac{2}{p}}}\right)+o(1) \\
& \geq m\left(\|u\|_{L^{p}}^{p}+\sum_{j} b_{j}\right)^{\frac{2}{p}}+o(1) \\
& =m\left(\int_{\mathbb{A}} d \nu\right)^{\frac{2}{p}}+o(1) \\
& =m^{p /(p-2)}+o(1) .
\end{aligned}
$$

By Lemma 35, $u=0$. Thus only one of $a_{j}$ is different from 0 , say $a_{1}=c>0$, $a_{j}=0, j=2,3, \cdots$. Thus $\left|\nabla u_{k}\right|^{2} d z=c \delta_{z_{1}}+o(1)$. Clearly $z_{1}=0$.

Similarly, let $\left\{w_{k}\right\}$ be the solutions of equation (1) in the interior flask domains $D_{k}$ as in Theorem 15, where $k>s_{0}$. Then we have the dynamic systems of $\left\{w_{k}\right\}$ as follows:

Theorem 37. Let $\bar{w}$ be as in Lemma 16. Then $w_{k} \rightarrow \bar{w}$ strongly in $H^{1}\left(\mathbb{R}^{N}\right)$ as $k \rightarrow \infty$.

Proof. Note that

$$
\left\{\begin{array}{l}
F\left(w_{k}\right)=\alpha\left(\mathbb{R}^{N}\right)+o(1), \\
F^{\prime}\left(w_{k}\right)=o(1) \text { as } k \rightarrow \infty
\end{array}\right.
$$

Note that with the same proof, Lemma 17 still holds in the entire space $\mathbb{R}^{N}$. By Lemma 17 in $\mathbb{R}^{N}$, we prove that there exist an integer $\ell \geq 0$, sequences $\left\{z_{k}^{i}\right\} \subset \mathbb{R}^{N}$ for $0 \leq i \leq \ell$, such that for some subsequence $\left\{w_{k}\right\}$, there are $w^{i} \in H^{1}\left(\mathbb{R}^{N}\right), w^{i}>0$ in $\mathbb{R}^{N}$ for $0 \leq i \leq \ell$, satisfying

$$
\left\{\begin{array}{c}
w_{k}(z)=w^{0}(z)+\left[w^{1}\left(z-z_{k}^{1}\right)+w^{2}\left(z-z_{k}^{2}\right)+\cdots\right. \\
\left.+w^{\ell}\left(z-z_{k}^{\ell}\right)\right]+o(1) \text { strongly in } H^{1}\left(\mathbb{R}^{N}\right) \\
-\triangle w^{i}+w^{i}=\left(w^{i}\right)^{p-1} \text { in } \mathbb{R}^{N}, 0 \leq i \leq \ell \\
F\left(w_{k}\right)=\sum_{i=0}^{\ell} F\left(w^{i}\right)+o(1) \text { as } k \rightarrow \infty
\end{array}\right.
$$

then by $F\left(w_{k}\right)=\alpha\left(\mathbb{R}^{N}\right)+o(1)$, we conclude that $w_{k}(z)=\bar{w}(z)+o(1)$ strongly in $H^{1}\left(\mathbb{R}^{N}\right)$.

\section{Multiple solutions of Perturbed EQuations}

In this section, we prove that there are two solutions of equation (2) in $\Omega$. 
Denote

$$
\begin{gathered}
c(p)=\left(\frac{1}{2}-\frac{1}{2(p-1)}\right)^{2}\left(\frac{p}{2(p-1)}\right)^{\frac{2}{p-2}} m^{\frac{p}{p-2}}, \\
\mathbf{Q}_{\rho}=\left\{u \in H_{0}^{1}(\Omega) \mid\|u\|_{H^{1}(\boldsymbol{\Omega})}<\rho\right\} .
\end{gathered}
$$

We have

Lemma 38. If $\|f\|_{L^{2}(\Omega)}^{2} \leq c(p)$, then there exists a positive constant $\rho_{0}$ such that $F_{f}(u) \geq 0$ for any $u \in \partial \mathbf{Q}_{\rho_{0}}$.

Proof. It is easy to see that the continuous function $k(t):[0,+\infty) \rightarrow \mathbb{R}$ defined by

$$
k(t)=\frac{1}{2} t-\frac{1}{p} m^{-\frac{p}{2}} t^{p-1},
$$

attains its maximum at $\rho_{0}=\left[\frac{p}{2(p-1)} m^{\frac{p}{2}}\right]^{\frac{1}{p-2}}$ and of the maximum value

$$
k\left(\rho_{0}\right)=\left(\frac{1}{2}-\frac{1}{2(p-1)}\right)\left(\frac{p}{2(p-1)}\right)^{\frac{1}{p-2}} m^{\frac{p}{2(p-2)}} .
$$

If $\|f\|_{L^{2}(\Omega)}^{2} \leq c(p)=k\left(\rho_{0}\right)^{2}$, we have, for all $u \in \partial \mathbf{Q}_{\rho_{0}}$,

$$
\begin{aligned}
F_{f}(u) & =\frac{1}{2} a(u)-\frac{1}{p} b(u)-\int_{\Omega} f u \\
& \geq \frac{1}{2}\|u\|_{H^{1}(\Omega)}^{2}-\frac{1}{p} m^{-\frac{p}{2}}\|u\|_{H^{1}(\Omega)}^{p}-\|f\|_{L^{2}(\Omega)}\|u\|_{H^{1}(\Omega)} \\
& \geq 0 .
\end{aligned}
$$

Remark 2. Let $\rho_{0}$ be as in Lemma 38. Then for $\epsilon>0$ small enough, there exists $\delta>0$ such that $F_{f}(u) \geq-\epsilon$ for any $u \in\left\{u \in H_{0}^{1}(\Omega) \mid \rho_{0}-\delta \leq\|u\|_{H^{1}(\Omega)} \leq \rho_{0}\right\}$.

Theorem 39. If $f \not \equiv 0, f \geq 0$ and $\|f\|_{L^{2}(\Omega)}^{2} \leq c(p)$, then there exists $u_{0} \in \mathbf{Q}_{\rho_{0}}$ such that $F_{f}\left(u_{0}\right)=\min \left\{F_{f}(u) \mid u \in \overline{\mathbf{Q}_{\rho_{0}}}\right\}<0$ and $u_{0}$ is a solution of equation (2) in $\Omega$.

Proof. Since $f \not \equiv 0$ and $f \geq 0$, we can choose a function $\varphi \in H_{0}^{1}(\Omega)$ such that $\int_{\Omega} f \varphi>0$. For $\lambda \in(0,+\infty)$, we have

$$
F_{f}(\lambda \varphi)=\frac{\lambda^{2}}{2} a(\varphi)-\frac{\lambda^{p}}{p} b(\varphi)-\lambda \int_{\Omega} f \varphi
$$

Then, for $\lambda$ small enough, $F_{f}(\lambda \varphi)<0$. Therefore, $\beta=\inf \left\{F_{f}(u) \mid u \in \overline{\mathbf{Q}_{\rho_{0}}}\right\}<0$. Clearly $\beta>-\infty$. By Remark 2 , there is $0<\rho_{1}<\rho_{0}$ such that $F_{f}(u) \geq \frac{\beta}{2}$ for $u \in\left\{u \in H_{0}^{1}(\Omega) \mid \rho_{1} \leq\|u\|_{H^{1}(\Omega)} \leq \rho_{0}\right\}$. By the Ekeland Variational Principle [10],

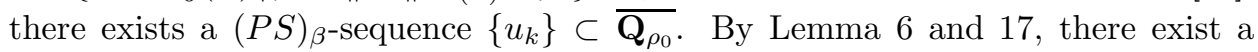
subsequence $\left\{u_{k}\right\}$, an integer $\ell \geq 0$, solutions $u^{i}$ of equation (1) in $\mathbb{S}, 1 \leq i \leq \ell$, and a solution $u_{0}$ in $\overline{\mathbf{Q}_{\rho_{0}}}$ of equation (2) such that $u_{k} \rightarrow u_{0}$ weakly in $H_{0}^{1}(\Omega)$ and $\beta=F_{f}\left(u_{0}\right)+\Sigma_{i=1}^{\ell} F\left(u^{i}\right)$. Note that $F\left(u^{i}\right) \geq F(\bar{u})>0$, where $\bar{u}$ is the ground state solution of Proposition 28, for $i=1,2, \cdots, \ell$. Since $u_{0} \in \overline{\mathbf{Q}_{\rho_{0}}}$, we have $F_{f}\left(u_{0}\right) \geq \beta$. We conclude that $\ell=0, F_{f}\left(u_{0}\right)=\beta$ and $F_{f}^{\prime}\left(u_{0}\right)=0$. 
We have obtained a solution of equation (2) in $\Omega$. In this section, we will assert that equation (2) in $\Omega$ admits another solution.

Let $\phi$ be as in Lemma 30 . Let $e_{N}=(0, \cdots, 1) \in \mathbb{R}^{N}$, denote

$$
\bar{u}_{\lambda}(z)=\phi(z) \bar{u}\left(z+\lambda e_{N}\right), \quad \lambda \in[0,+\infty),
$$

where $\bar{u}$ is the ground state solution of Proposition 28 .

Lemma 40. If $f \geq 0, f \not \equiv 0,\|f\|_{L^{2}(\Omega)}^{2} \leq c(p)$ and there exist positive constants $\epsilon, c$ such that $0 \leq f(z) \leq c \exp \left(-\sqrt{1+\lambda_{1}+\epsilon}|y|\right)$, for all $z \in \Omega$. Then there exists $\lambda_{0}>0$ such that, for $\lambda \geq \lambda_{0}$, we have

$$
\sup _{t \geq 0} F_{f}\left(u_{0}+t \bar{u}_{\lambda}\right)<F_{f}\left(u_{0}\right)+F(\bar{u}),
$$

where $u_{0}$ is the local minimum in Theorem 39.

Proof. Since $F_{f}$ is continuous in $H_{0}^{1}(\Omega)$, there exists $t_{0}>0$ such that for $0 \leq t<t_{0}$,

$$
F_{f}\left(u_{0}+t \bar{u}_{\lambda}\right)<F_{f}\left(u_{0}\right)+F(\bar{u}) \quad \text { for all } \lambda \in[0,+\infty) .
$$

Then we only need to verify the inequality

$$
\sup _{t \geq t_{0}} F_{f}\left(u_{0}+t \bar{u}_{\lambda}\right)<F_{f}\left(u_{0}\right)+F(\bar{u}),
$$

for $\lambda$ large enough. First, we observe that if $r, o$ are arbitrary nonnegative, then there exists a constant $c>0$ independent of $r$ and $o$ such that

$$
(r+o)^{p} \geq r^{p}+o^{p}+p\left(r^{p-1} o+r o^{p-1}\right)-c r^{\frac{p}{2}} o^{\frac{p}{2}} .
$$

Hence we get

$$
\begin{aligned}
\int_{\Omega}\left(u_{0}+t \bar{u}_{\lambda}\right)^{p} d x & \geq \int_{\Omega}\left[u_{0}^{p}+\left(t \bar{u}_{\lambda}\right)^{p}+p\left(t u_{0}^{p-1} \bar{u}_{\lambda}+\left(t \bar{u}_{\lambda}\right)^{p-1} u_{0}\right)\right] d x \\
& -c \int_{\Omega} u_{0}^{\frac{p}{2}}\left(t \bar{u}_{\lambda}\right)^{\frac{p}{2}} d x .
\end{aligned}
$$

We deduce for $t \geq t_{0}$,

$$
\begin{aligned}
F_{f}\left(u_{0}+t \bar{u}_{\lambda}\right) \\
=\frac{1}{2} \int_{\Omega}\left(\left|\nabla\left(u_{0}+t \bar{u}_{\lambda}\right)\right|^{2}+\left|u_{0}+t \bar{u}_{\lambda}\right|^{2}\right) \\
-\frac{1}{p} \int_{\Omega}\left(u_{0}+t \bar{u}_{\lambda}\right)^{p}-\int_{\Omega} f(z)\left(u_{0}+t \bar{u}_{\lambda}\right) \\
\leq F_{f}\left(u_{0}\right)+F(\bar{u})-t^{\frac{p}{2}}\left(t_{0}^{\frac{p-2}{2}} \int_{\Omega} \bar{u}_{\lambda}^{p-1} u_{0}-c \int_{\Omega} u_{0}^{\frac{p}{2}} \bar{u}_{\lambda}^{\frac{p}{2}}\right) .
\end{aligned}
$$

We choose $\delta>0$ small enough, such that

$$
\sqrt{1+\lambda_{1}+\delta}<\frac{p}{2} \sqrt{1+\lambda_{1}-\delta}
$$

Applying Lemma 27, we find that there exist positive constants $c_{1}, c_{2}$ such that, for all $\lambda \in[0,+\infty)$,

$$
\begin{aligned}
& \int_{\Omega} \bar{u}_{\lambda}^{p-1} u_{0} \geq c_{1} \int_{\Omega_{1}} \bar{u}_{\lambda}^{p-1} e^{-\sqrt{1+\lambda_{1}+\delta}|y|} d z, \\
& \int_{\Omega} u_{0}^{\frac{p}{2}} \bar{u}_{\lambda}^{\frac{p}{2}} \leq c_{2} \int_{\Omega} \bar{u}_{\lambda}^{\frac{p}{2}} e^{-\sqrt{1+\lambda_{1}-\delta} \frac{p}{2}|y|} d z .
\end{aligned}
$$


Now, let $z_{N}=\left\langle z, e_{N}\right\rangle$, we deduce, as $\lambda \rightarrow \infty$, where $\Omega_{1}$ is as in Lemma 27,

$$
\begin{aligned}
\int_{\Omega} \bar{u}_{\lambda}^{p-1} u_{0} & \geq c_{1} \int_{\Omega_{1}} \bar{u}_{\lambda}^{p-1} e^{-\sqrt{1+\lambda_{1}+\delta}|y|} d z \\
& =c_{1} \int_{\Omega_{1}} \bar{u}^{p-1}(z) e^{-\sqrt{1+\lambda_{1}+\delta}\left|y-\lambda e_{N}\right|} d z \\
& =c_{1}\left(\int_{\Omega_{1}} \bar{u}^{p-1}(z) e^{z_{N} \sqrt{1+\lambda_{1}+\delta}} d z+o(1)\right) e^{-\lambda \sqrt{1+\lambda_{1}+\delta}}
\end{aligned}
$$

and

$$
\begin{aligned}
\int_{\Omega} u_{0}^{\frac{p}{2}} \bar{u}_{\lambda}^{\frac{p}{2}} & \leq c_{2} \int_{\Omega} \bar{u}_{\lambda}^{\frac{p}{2}} e^{-\frac{p}{2} \sqrt{1+\lambda_{1}-\delta}|y|} d z \\
& =c_{2} \int_{\Omega} \bar{u}_{\lambda}^{\frac{p}{2}}(z) e^{-\frac{p}{2} \sqrt{1+\lambda_{1}-\delta}\left|y-\lambda e_{N}\right|} d z \\
& =c_{2}\left(\int_{\Omega} \bar{u}_{\lambda}^{\frac{p}{2}}(z) e^{\frac{p}{2} z_{N} \sqrt{1+\lambda_{1}-\delta}} d z+o(1)\right) e^{-\frac{p}{2} \lambda \sqrt{1+\lambda_{1}-\delta}} .
\end{aligned}
$$

Then we find that there exists $\lambda_{0}>0$ such that, for $\lambda \geq \lambda_{0}$,

$$
t_{0}^{\frac{p-2}{2}} \int_{\Omega} \bar{u}_{\lambda}^{p-1} u_{0}-c \int_{\Omega} u_{0}^{\frac{p}{2}} \bar{u}_{\lambda}^{\frac{p}{2}}>0
$$

Therefore, for $\lambda \geq \lambda_{0}$,

$$
\sup _{t \geq 0} F_{f}\left(u_{0}+t \bar{u}_{\lambda}\right)<F_{f}\left(u_{0}\right)+F(\bar{u}) .
$$

Theorem 41. Let $f \geq 0, f \not \equiv 0,\|f\|_{L^{2}(\Omega)}^{2} \leq c(p)$ and there exist positive constants $\epsilon, c$ such that $0 \leq f(z) \leq c \exp \left(-\sqrt{1+\lambda_{1}+\epsilon}|y|\right)$ for all $z \in \Omega$. Then equation (2) has at least two positive solutions.

Proof. By Lemma 40, there exists $\rho_{0}>0$ such that

$$
F_{f}(u) \geq 0 \quad \text { for } u \in \partial \mathbf{Q}_{\rho_{0}} .
$$

Fix $\lambda \geq \lambda_{0}$ such that

$$
\sup _{t \geq 0} F_{f}\left(u_{0}+t \bar{u}_{\lambda}\right)<F_{f}\left(u_{0}\right)+F(\bar{u}) .
$$

From (3) and (4), it is easy to see that there exists $t_{1}>0$ such that $F_{f}\left(u_{0}+t \bar{u}_{\lambda}\right)<0$ for $t \geq t_{1}$ and $u_{0}+t_{1} \bar{u}_{\lambda} \notin \overline{\mathbf{Q}_{\rho_{0}}}$. Set

$$
\begin{gathered}
\Gamma=\left\{\kappa \in C\left([0,1], H_{0}^{1}(\Omega)\right) \mid \kappa(0)=u_{0}, \kappa(1)=u_{0}+t_{1} \bar{u}_{\lambda}\right\}, \\
c=\inf _{\kappa \in \Gamma} \max _{s \in[0,1]} F_{f}(\kappa(s)) .
\end{gathered}
$$

By Lemma 38 and 40, we have

$$
0 \leq c=\inf _{\kappa \in \Gamma} \max _{s \in[0,1]} F_{f}(\kappa(s))<F_{f}\left(u_{0}\right)+F(\bar{u}) .
$$

We conclude that $F_{f}$ satisfies the Mountain Pass hypothesis, so there exists a $(P S)_{c}$-sequence $\left\{u_{k}\right\}$ in $H_{0}^{1}(\Omega)$ such that

$$
\left\{\begin{array}{l}
F_{f}\left(u_{k}\right) \rightarrow c, \\
F_{f}^{\prime}\left(u_{k}\right) \rightarrow 0 \text { strongly in } H^{-1}(\Omega) .
\end{array}\right.
$$


By Lemma 17, there exist a subsequence $\left\{u_{k}\right\}$, nonnegative integer $\ell$, positive solutions $u^{i}, 1 \leq i \leq \ell$, of equation (1) in $\mathbb{S}$, and a positive solution $u^{0}$ of equation (2) in $\Omega$ such that

$$
c=F_{f}\left(u^{0}\right)+\sum_{i=1}^{\ell} F\left(u^{i}\right) .
$$

Next, we prove $u^{0}$ is another positive solution of equation (2) in $\Omega$. As a matter of fact, we have

$$
F_{f}\left(u_{0}\right)<c=F_{f}\left(u^{0}\right)+\lambda F(\bar{u})<F_{f}\left(u_{0}\right)+F(\bar{u}),
$$

where $\lambda \geq 1$ if $\ell \geq 1, \lambda=0$ if $\ell=0$.

(1) If $\ell=0$, then $F_{f}\left(u_{0}\right)<F_{f}\left(u^{0}\right)$.

(2) If $\ell \geq 1$, then $F_{f}\left(u^{0}\right)<F_{f}\left(u_{0}\right)$.

\section{ACKNOWLEDGMENT}

The author is grateful to the referee whose valuable comments helped to improve the contents of this paper.

\section{REFERENCES}

1. R.A. Adams, Sobolev Spaces, Academic Press, New York, 1975. MR 56:9247

2. V. Benci and G. Cerami, Positive solutions of some nonlinear elliptic problems in exterior domains, Arch. Rational Mech. Anal., 99 (1987), 282-300. MR 88f:35014

3. H. Berestycki and P. L. Lions, Nonlinear scalar field equations. I. Existence of ground state, Arch. Rat. Mech. Anal., 82 (1983), 313-345. MR 84h:35054a

4. H. Brezis and T. Kato, Remarks on the Schrodinger operator with singular complex potentials, J. Math. Pures Appl., 58 (1979), 137-151. MR 80i:35135

5. H. Brezis and L. Nirenberg, Remarks on finding critical points, Comm. on Pure and Appl. Math., 44 (1991), 939-963. MR 92i:58032

6. K. J. Chen, K. C. Chen, and H. C. Wang, Symmetry of positive solutions of semilinear elliptic equations in infinite strip domains, J. Differential Equations, 148 (1998), 1-8. CMP 98:16

7. K. J. Chen, C. S. Lee, and H. C. Wang, Semilinear elliptic problems in interior and exterior flask domains, commun. Appl. Nonlinear Anal., 6 (1999).

8. J. M. Coron, Topologie et cas limite des injections de Sobolev, C. R. Acad. Sci. Paris Ser. I Math., 299 (1984), 209-212. MR 86b:35059

9. E. N. Dancer, On the influence of domain shape on the existence of large solutions of some superlinear problems, Math. Ann., 285 (1989), 647-669. MR 91h:35122

10. I. Ekeland, Nonconvex minimization problems, Bull. Amer. Math. Soc., 1 (1979), 443-474. MR 80h:49007

11. M. J. Esteban and P. L. Lions, Existence and non-existence results for semilinear elliptic problems in unbounded domains, Proc. Royal Soc. Edinburgh, 93A (1982), 1-14. MR 85e:35047]

12. B. Gidas, W. M. Ni and L. Nirenburg, Symmetry of positive solutions of nonlinear elliptic equations in $\mathbb{R}^{N}$, Adv. in Math. Suppl. Stud., 7A (1981), 369-402. MR 84a:35083

13. D. Gilbarg and N.S. Trudinger, Elliptic Partial Differential Equations of Second order, Springer-Verlag, New York, 1983. MR 86c:35035

14. T. S. Hsu and H. C. Wang, A perturbation result of semilinear elliptic equations in exterior strip domains, Proc. Royal Soc. Edinburgh, 127A (1997), 983-1004. MR 98h:35063

15. M. K. Kwong, Uniqueness of positive solutions of $\Delta u-u+u^{p}=0$ in $\mathbb{R}^{N}$, Arch. Rat. Mech. Anal., 105 (1989), 243-266. MR 90d:35015

16. W.C. Lien, S.Y. Tzeng and H.C. Wang, Existence of solutions of semilinear elliptic problems on unbounded domains, Differential Integral Equations 6 (1993), 1281-1298. MR 94h:35061 
17. J. L. Lions and E. Zuazua, Approximate controllability of a hydro-elastic coupled system, ESMAIM Contrôle Optim. Calc. Var. 1 (1995/96), 1-15. MR 97a:93012

18. P. L. Lions, The concentration-compactness principle in the calculus of variations, the limit case, Rev. Mat. Iberoamericana, 1 (1985), Part 1, 45-121, Part 2, 145-201. MR 87j:49012. MR 87c:49007

19. S. I. Pohozaev, Eigenfunctions of the equation $\Delta u+\lambda f(u)=0$, Soviet Math. Dokl., 6 (1965), 1408-1411.

20. C. A. Stuart, Bifurcation in $L^{p}\left(\mathbb{R}^{N}\right)$ for a semilinear elliptic equations, Proc. London Math. Soc., 45 (1982), 169-192.

21. X.P. Zhu, A perturbation result on positive entire solutions of a semilinear elliptic equations, J. Differential Equations, 92 (1991), 163-178. MR 92g:35019

Department of Mathematics, National Tsing Hua University, Hsinchu, Taiwan

E-mail address: hwang@math.nthu.edu.tw 